\title{
Universal screening of high-risk neonates, parents, and staff at a neonatal intensive care unit during the SARS-CoV-2 pandemic
}

\author{
Maria Elena Cavicchiolo ${ }^{1}$ (D) Daniele Trevisanuto ${ }^{1} \cdot$ Elisabetta Lolli $^{1} \cdot$ Veronica Mardegan $^{1} \cdot$ Anna Maria Saieva $^{2}$. \\ Elisa Franchin ${ }^{3} \cdot$ Mario Plebani $^{4} \cdot$ Daniele Donato $^{2} \cdot$ Eugenio Baraldi $^{1}$
}

Received: 15 June 2020 / Revised: 27 July 2020 / Accepted: 30 July 2020 / Published online: 7 August 2020

(C) Springer-Verlag GmbH Germany, part of Springer Nature 2020

\begin{abstract}
Since February 21, 2020, SARS-CoV-2 has spread exponentially worldwide. Neonatal patients needing intensive care are considered a vulnerable population. To report the results of a policy based on multi-timepoint surveillance for SARS-CoV-2 of all neonates admitted to the neonatal intensive care unit (NICU), their parents, and all healthcare providers in a part of Italy with a high prevalence of the infection. Observational study conducted from 21 February to 21 April 2020. Intervention consisted of (a) parental triage on arrival at the neonatal ward; (b) universal testing with nasopharyngeal swabs and blood testing for SARSCoV-2 IgM and IgG antibodies; (c) use of continuous personal protective equipment at the NICU by parents and staff. A total of 6726 triage procedures were performed on 114 parents, and 954 nasopharyngeal swabs were collected from 226 individuals. Five $(2.2 \%)$ asymptomatic individuals ( 2 parents and 3 healthcare providers) tested positive on nasopharyngeal swabs and were kept isolated for 14 days. Of 75 admitted newborn, no one tested positive on nasopharyngeal swabs or antibody tests. Three parents presented with fever or flu-like symptoms at triage; they tested negative on swabs.

Conclusion: With universal screening of neonates, parents, and staff, there were no cases of SARS-CoV-2 infection among the neonates admitted to a NICU in an area with a high incidence of SARS-CoV-2. Our experience could be usefully compared with other strategies with a view to developing future evidence-based guidelines for managing high-risk neonates in case of new epidemics.
\end{abstract}

What is Known:

- The novel coronavirus named SARS-CoV-2 has since spread worldwide at a remarkable rate, with more than 2.5 million confirmed cases.

- Pediatric population may be less affected from COVID-19 than adult population but infants and newborn babies seem to be more vulnerable to SARS-CoV-2 infection.

What is New:

- Using an approach based on triage; testing with nasopharyngeal swabs and serology; and use of personal protective equipment, there were no cases of SARS-CoV-2 infection among neonates in a NICU in a high incidence of SARS-CoV-2 area.

- Positive and asymptomatic individuals were identified and isolated early allowing the containment of infection's spread among healthcare providers and parents.

Keywords Neonatology $\cdot$ Newborn infant $\cdot$ Neonatal intensive care unit $\cdot$ Preterm infant $\cdot$ SARS-CoV-2 $\cdot$ Covid-19 $\cdot$ Very low birth weight infant

Maria Elena Cavicchiolo and Daniele Trevisanuto contributed equally to this work.

Communicated by Daniele De Luca

Eugenio Baraldi

eugenio.baraldi@unipd.it

1 Department of Women's and Children's Health, Neonatal Intensive Care Unit, Padova University Hospital, Padova, Italy

2 Padova University Hospital, Padova, Italy
3 Department of Molecular Medicine, Microbiology and Virology Unit, Padova University Hospital, Padova, Italy

4 Department of Clinical Chemistry and Laboratory Medicine, Department of Medicine, Padova University Hospital, Padova, Italy 


\section{Abbreviation \\ AAP American Academy of Pediatrics \\ CDC Centers for Disease Control and Prevention \\ NETS Neonatal emergency transport system \\ NICU Neonatal intensive care unit \\ PPE Personal protective equipment \\ RT-PCR Real-time polymerase chain reaction \\ VLBWI Very low birth weight infants}

\section{Introduction}

The novel coronavirus named SARS-CoV-2, responsible for Covid-19, was first identified in December 2019 in Wuhan, the capital of China's Hubei province. It has since spread worldwide at a remarkable rate, with more than 5.5 million confirmed cases and 350 thousand deaths reported by 27 March 2020, including over 30 thousand deaths in Italy [1].

SARS-CoV-2 is a virus with a high rate of transmission among humans. Studies have shown that a significant proportion of individuals with SARS-CoV-2 are asymptomatic and can transmit the virus to others in close contact, via tiny droplets produced by coughing, sneezing, or talking [2, 3]. People may also become infected by touching a contaminated surface and then touching their eyes, nose, or mouth (the virus can survive on surfaces for up to $72 \mathrm{~h}$ ) [4]. While older people and adults with underlying health conditions seem to be at greatest risk of developing severe SARS-CoV-2-related illness, young children and newborn babies are also susceptible to the infection. Pneumonia has been described in a few neonates born to mothers with Covid-19 [5, 6]. Vertical transmission has also been demonstrated [7] but rarely reported [8].

On precautionary grounds, preterm newborns are considered a vulnerable population with an immature immune system, and consequently at greater risk of severe infections. Extremely low birth weight infants (ELBWI) are particularly susceptible to respiratory problems that may contribute to the development of bronchopulmonary dysplasia. In the past, human coronaviruses have been known to cause serious respiratory infections in preterm infants [9] and in immunocompromised children [10]. For these reasons, SARS-CoV-2 is an important concern for healthcare providers caring for preterm infants.

Discordant international recommendations have become available on how to manage neonates born to SARS-CoV-2positive mothers in recent weeks [11-13], but evidence-based guidelines are still lacking and pediatric international registries are still ongoing [14].

In Italy, one of the regions most severely affected by the new virus is Veneto, where Padua's University Hospital is the Covid-19 referral center. Right from the start of the pandemic, the Veneto region's approach was based on widespread screening of the population [15].
The aim of this report is to describe the results of a policy based on epidemic multi-timepoint surveillance and screening for SARS-CoV-2 of all neonates admitted to our neonatal ward in Padua, their parents, and all healthcare providers, in a part of Italy with a high prevalence of the infection.

\section{Methods}

\section{Setting}

The Pediatric Department at Padua University Hospital is a tertiary referral center for eastern Veneto, where about 3000 deliveries occur every year. The NICU is a 35 -bed facility comprising pods containing four to six cots each for a total of 20 intensive care beds, with two isolated negative-pressure rooms and 15 beds for high-dependency patients. The NICU handles 400 hospitalizations a year, including 110 very low birth weight infants (VLBWI). The staff consists of 59 nurses, 12 neonatologists, 14 pediatric residents, and 6 consultants, who also guarantee a neonatal emergency transport system (NETS) for the eastern Veneto area, with about 180-200 emergency transports and 70 return transports every year.

\section{Population}

All neonates admitted to the NICU from February 21 to April 21,2020 , irrespective of any respiratory symptoms, and all staff and parents were included in the study.

The study protocol complied with the Helsinki Declaration and was notified to the Institutional Ethical Committee in Padua (ref. number 00270821). Parents signed an informed consent form before the first specimen was collected.

\section{Intervention}

We previously reported on the measures taken at the NICU to prevent or contain SARS-CoV-2 infections in sick neonates and preterm babies [16]. Briefly, the intervention focused on three aspects: (a) triage for parents on arrival at the neonatal ward, and training on measures for preventing infections; (b) universal testing with weekly nasopharyngeal swabs and quantitative polymerase chain reaction tests to detect SARS$\mathrm{CoV}-2$ infection in all neonates, parents, and healthcare staff, and testing for SARS-CoV-2 IgG and IgM antibodies in neonates; (c) continuous use of personal protective equipment (PPE) during neonatal care and at all times in the NICU.

\section{a. Triage for parents}

A triage system was established for all parents of newborns admitted to the NICU. Their body temperature was measured, and information was collected on their health status, contacts 
with suspected or known cases of Covid-19 in the previous 2 weeks, and any influenza-like symptoms.

To avoid overcrowding, parental visits were restricted to $1 \mathrm{~h}$ a day, and only one parent per baby could be admitted at scheduled times. All parents were asked to wear masks, gloves, and disposable clothing. Use of mobile phones was not allowed. All close contacts, such as kangaroo mother care and holding the baby, were suspended. A video calling service was activated for parents in quarantine who could not visit their children.

\section{b. Nasopharyngeal swabs and serological assays}

Nasopharyngeal swabs were collected and placed in viral transport medium for RNA detection (typically real-time polymerase chain reaction (RT-PCR)) to diagnose SARS-CoV-2 according to the Centers for Disease Control and Prevention (CDC) guidelines [17].

Individuals testing negative were re-tested once a week. Those testing positive were quarantined and supervised by an infectious diseases specialist. High-risk patients (born to mothers with suspected or confirmed SARS-CoV-2 infection) or positive patients were tested every $24 \mathrm{~h}$ for the first 3 days from birth or the time of coming into contact with the virus.

Specimens were analyzed at the certified microbiological laboratory of Padua University Hospital. Results were available after $12-48 \mathrm{~h}$.

From April 7 onwards, all hospitalized patients were tested for SARS-CoV-2 IgG and IgM antibodies using the

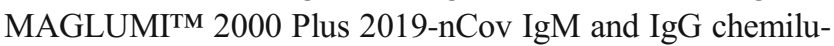
minescence immunoassay (Snibe, Shenzhen, China) [18]. Results were available in 24-48 h. Neonates newly admitted to the NICU were tested for antibodies within the first day of life; those already hospitalized were tested as soon as the test became available. Only neonates at risk of SARS-CoV-2 infection with a positive maternal history were tested at birth and at 14 days of life. Antibody title was also evaluated for health care providers and for subjects with a positive swab.

\section{c. Isolation and quarantine zone}

All newborns were kept isolated in closed isolettes. Infants born to mothers testing positive for SARS-CoV-2 were kept isolated in a dedicated NICU negative pressure "quarantine zone," where parents were not allowed. After 14 days in quarantine and three consecutive negative nasopharyngeal swabs, patients were moved to the standard ward.

\section{d. Breastfeeding}

In accordance with AAP guidelines [12], newborns were kept separate from suspected or known COVID-19-positive mothers. LBWI and/or those born at a gestational age of less than 29 weeks were fed with pasteurized donor's milk. SARSCoV-2-positive mothers were urged to pump milk to maintain milk supply.

\section{e. NICU and NETS reorganization}

Staff wore surgical masks at the NICU and the importance of hand hygiene, based on frequent hand-washing and the use of alcoholic disinfectants, was stressed. In the quarantine zone, staff wore PPE (N95 respirators, double gloves, eye protection, and gowns). Staff were given training on the correct procedure for donning and doffing PPE.

Written educational material was given to healthcare providers and parents to help prevent viral transmission. The instructions included staying at home, keeping their distance from others, frequent hand hygiene, disinfecting objects and surfaces, and reporting any symptoms.

A specific protocol was developed for the NETS to recognize high-risk cases promptly, decide the safest way to bring the newborn to the referral hospital, and ensure their safe stabilization in transit [19].

\section{f. Psychological support for families and NICU staff}

Psychological support was offered and provided to parents and NICU staff.

\section{Statistical analysis}

Data were collected by reviewing all the original medical records as well as the relevant clinical electronic databases. The main results are reported as descriptive analyses. Data were entered and analyzed in an electronic database (Microsoft Excel, version 16.35, 2020).

\section{Results}

Sixty-five patients were admitted to the neonatal ward between February 21 and April 21, 2020, and 23 of them were VLBWI. Thirty-six newborn (60\%) needed intensive care, and 8 of them $(22 \%)$ needed mechanical ventilation, while the remainder were treated with noninvasive respiratory support.

We performed 6726 triage procedures on 114 parents and 112 healthcare providers. Three parents presented with fever or flu-like symptoms. They were urgently tested for SARS$\mathrm{CoV}-2$ and refused entry until the infection was ruled out. They all tested negative. In 8 weeks of surveillance, 954 nasopharyngeal swabs were collected from 226 individuals (Fig. 1). Two parents and three healthcare providers $(2.2 \%)$ tested positive and were kept isolated for 14 days (Table 1). They were all asymptomatic. No seroconversion 


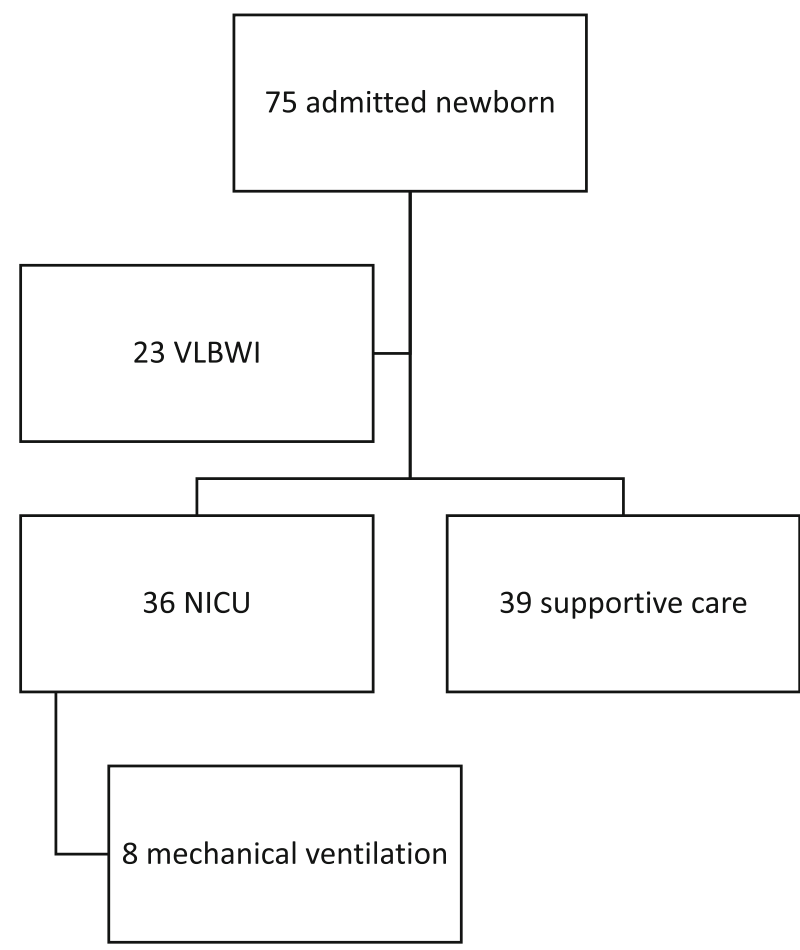

Fig. 1 Flowcharts of the main results of the study

occurred also in positive subjects. No newborn tested positive for SARS-CoV-2. Serology was negative in all neonates admitted.

During the study period, two SARS-CoV-2-positive women delivered at Padua Hospital. Three high-risk newborns were kept in the quarantine zone: one was born to a mother with severe SARS-CoV-2 respiratory distress needing mechanical ventilation; two had a history of close contact with someone infected. Table 2 shows their clinical characteristics. These high-risk newborns were tested every $24 \mathrm{~h}$ for the first 3 days. As they tested negative, they were moved on day 14 to a standard room and tested for SARS-CoV-2 every week. No seroconversions occurred.

No newborn with ascertained or suspected SARS-CoV-2 infection were brought in by the NETS service during the pandemic period analyzed.

Parents and all healthcare providers showed strong compliance and great motivation in respecting the new organization, social distancing, and hygiene rules adopted for the NICU. All parents must wear PPE (facemasks, gloves, and disposable clothing). Two parents asked for psychological support.

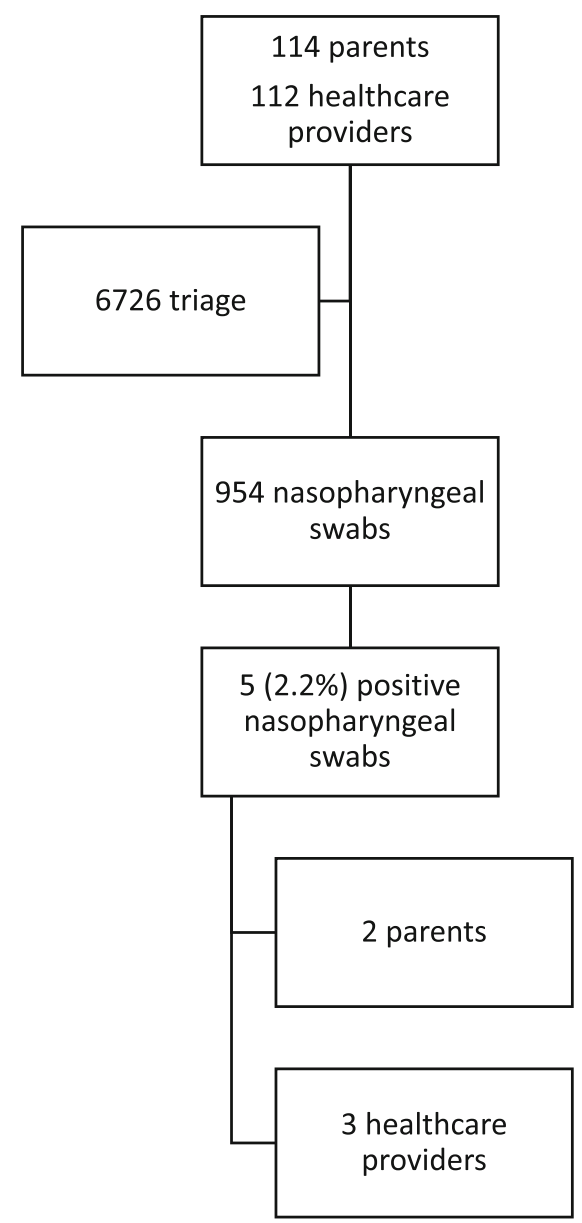

\section{Discussion}

To our knowledge, this is the first study reporting on the universal screening of preterm neonates, parents, and staff at a NICU in an area with high incidence of SARS-CoV-2 infection. With universal SARS-CoV-2 testing, $2.2 \%$ of our selected population tested positive during the time of the epidemic and they were all asymptomatic. No neonates tested positive on either quantitative RT-PCR or serology, not even those born to COVID-19-positive mothers.

In Italy, the first outbreak of SARS-CoV-2 was reported in the municipality of Vo', a few miles from Padova. All 3300 residents were tested on the day the first case was detected (21 February 2020), and $2.6 \%$ of them tested positive. More than $40 \%$ of the people infected were completely asymptomatic [15]. With aggressive testing, the quarantining of people found positive for SARS-CoV-2, and the total isolation of the town, the epidemic was extinguished. There is growing evidence to indicate that asymptomatic individuals are an important unwitting source of contagion [2, 3, 20], and around $50-60 \%$ of individuals testing positive for SARS-CoV-2 are asymptomatic or pre-symptomatic [21]. 
Table 1 Clinical characteristics of positive real-time polymerase chain reaction SARS-CoV-2 subjects

\begin{tabular}{|c|c|c|c|c|c|}
\hline Age, sex & Classification & Nasopharyngeal swab & Covid-19 symptoms & $\begin{array}{l}\text { Contact with Covid-19-positive } \\
\text { individuals }\end{array}$ & Management \\
\hline 35 years, $F$ & Mother & Positive (13/03/2020) & None & No & Quarantine at home \\
\hline 38 years, $\mathrm{M}$ & Father & Positive (01/03/2020) & None & $\begin{array}{l}\text { Yes, close contact with a positive } \\
\text { asymptomatic subject }\end{array}$ & Quarantine at home \\
\hline 55 years, $\mathrm{F}$ & Nurse & Positive (17/03/2020) & None & $\begin{array}{l}\text { Yes, living with her positive } \\
\text { symptomatic parents }\end{array}$ & Quarantine at home \\
\hline 29 years, $\mathrm{F}$ & Resident & Positive (31/03/2020) & None & No & Quarantine at home \\
\hline 37 years, $F$ & Physiotherapist & Positive $(31 / 03 / 2020)$ & None & No & Quarantine at home \\
\hline
\end{tabular}

As neonates admitted to the NICU, especially LBWIs, are a highly vulnerable population, we opted for an early heightened surveillance, with universal testing of all newborns admitted to the NICU, their parents, and our staff, in line with the approach taken for the Veneto region generally. Interestingly, the incidence of SARS-CoV-2 infection in our selected sample was similar to the figure reported by Lavezzo et al., in the population of Vo [15]. An approach based on universal screening has proved capable of containing the virus's transmission in the general population [15]. Although no firm conclusions can be drawn from our experience, we would argue that promptly identifying positive asymptomatic cases on arrival at the neonatal ward could have contributed in containing the impact of the infection among our parents, staff, and neonates.

Testing all admitted newborns, their parents, and healthcare providers might be questionable due to the increased burden on human and economic resources [22]. The additional costs during the study period can be estimated as the following: (1) nurse availability for triage: $1.5 \mathrm{~h} /$ day for a total of $84 \mathrm{~h}$ in 8 weeks; (2) 126\% increased use of disposable PPE in respect to the same period of the previous year; (c) 954 RT-PCR tests performed.

Even if vertical transmission seems unlikely, it still cannot be completely ruled out $[7,8,23,24]$. The main source of SARS-CoV-2 infection in neonates is due to close contact with SARS-CoV-2-positive caregivers [5, 23-25], as reported for children [26]. For now, only a few cases of SARS-CoV-2 infections have been described, including anecdotal neonatal deaths $[5,23,24,27]$. The effect of SARSCoV-2 in a high-risk population should not be underestimated, bearing in mind that, years ago, another coronavirus (HCoV-229E) infected more than $50 \%$ of the preterm newborn at a NICU in France, in association with a high rate of infection among staff [8]. Moreover, no vaccine or appropriate antiviral treatment for SARS-CoV-2 is available as yet; therefore, prevention seems to be the most effective strategy to fight the epidemic. All these factors support a strategy to maximize the prevention measures for parents accessing the NICU.

Table 2 Clinical characteristics of Covid-19 high-risk newborns

\begin{tabular}{|c|c|c|c|c|c|c|}
\hline Age, sex & $\begin{array}{l}\text { Birth } \\
\text { weight }\end{array}$ & History & $\begin{array}{l}\text { Nasopharyngeal } \\
\text { swab }\end{array}$ & $\begin{array}{l}\text { Serological } \\
\text { test }\end{array}$ & Diagnosis & Management \\
\hline $\begin{array}{l}25+3 \text { weeks' } \\
\text { gestation, } \\
\text { male }\end{array}$ & $840 \mathrm{~g}$ & $\begin{array}{l}\text { Born from mother with } \\
\text { SARS-CoV-2 severe respirato- } \\
\text { ry distress }\end{array}$ & Negative & Negative & $\begin{array}{l}\text { Prematurity } \\
\text { RDS (intubation and surfactant at } \\
\text { birth, mechanical ventilation for } \\
48 \mathrm{~h} \text { ) } \\
\text { Patent ductus arteriosus treated with } \\
\text { ibuprofen }\end{array}$ & $\begin{array}{l}\text { Quarantine zone } \\
\text { Nasopharyngeal } \\
\text { swabs } \\
\text { Serological tests } \\
\text { No parental visit }\end{array}$ \\
\hline $\begin{array}{l}40+4 \text { weeks' } \\
\text { gestation, } \\
\text { female }\end{array}$ & $3340 \mathrm{~g}$ & $\begin{array}{l}\text { Breastfed while mother was } \\
\text { asymptomatic SARS-CoV-2 } \\
\text { positive }\end{array}$ & Negative & Negative & $\begin{array}{l}\text { Congenital heart malformation } \\
\text { (double outlet right ventricle) }\end{array}$ & $\begin{array}{l}\text { Quarantine zone } \\
\text { Nasopharyngeal } \\
\text { swabs } \\
\text { Serological tests } \\
\text { No parental visit }\end{array}$ \\
\hline $\begin{array}{l}35+3 \text { weeks' } \\
\text { gestation, } \\
\text { female }\end{array}$ & $2890 \mathrm{~g}$ & $\begin{array}{l}\text { Strict contact with an unaware } \\
\text { Covid-19-positive consultant }\end{array}$ & Negative & Negative & Duodenal perforation & $\begin{array}{l}\text { Quarantine zone } \\
\text { Nasopharyngeal } \\
\text { swabs } \\
\text { Serological tests } \\
\text { No parental visit }\end{array}$ \\
\hline
\end{tabular}

$R D S$ respiratory distress syndrome 
Serology performed in addition to swabs has been identified by the FDA and WHO as a potentially useful method diagnosing SARS-CoV-2 [28, 29]. Negative results of both nasopharyngeal swabs and antibody tests enabled us to rule out the infection in all infants at our unit with almost absolute certainty.

We adopted an approach based on three pillars (triage and education; testing with nasopharyngeal swabs and serology; and the use of PPE), but for now, it is impossible to say what weight each of these measures had on the final outcome.

Parents appeared to take a positive attitude to our restrictive policy. Measuring the psychological impact of the limitations imposed on their physical contact with their babies is beyond the scope of the present study but should be assessed in future research.

This study has some limitations. First, it reports a strategy to contain the diffusion of SARS-CoV-2 at a NICU, but it cannot demonstrate its superiority over any other policy based on a different approach and it cannot be applied in a limitedresource setting. Second, our model cannot be generalized to all neonatal wards because the strategy described here was designed for a setting with a pod-based arrangement and might not be appropriate for other settings and NICUs with different structural layout (i.e., single-family rooms). Finally, as we did not examine placenta, amniotic fluid, and cord blood for SARS-CoV-2 of all newborns, some positivity may have been missed.

In conclusion, the present study describes the findings of the universal screening of neonates, parents, and staff at a neonatal ward in an area with a high incidence of SARSCoV-2. No cases of SARS-CoV-2 infection occurred among the neonates admitted. Positive asymptomatic individuals ( $2 \%$ of screened) were identified and isolated early allowing the containment of infection's spread in the NICU. The lesson learned from our experience needs to be compared with other policies adopted elsewhere at units caring for high-risk neonates during the SARS-CoV-2 pandemic. Such comparisons could be useful for the purpose of developing evidence-based guidelines for the future management of high-risk neonates in case of new Covid-19 pandemic.

Acknowledgments We gratefully acknowledge all NICU staff for their tremendous support during this challenging period. Physicians: Bonadies Luca, Calgaro Serena, Doglioni Nicoletta, Magarotto Mariella, Nardo Daniel, Piva Daniele, Priante Elena, Salvadori Sabrina, Trafojer Ursula Maria Theresia, Verlato Giovanna. Residents: Borellini Martina, Bua Benedetta, Casarin Elisa, Congedi Sabrina, Contin Annaelena, D’Onofrio Gianluca, Faggian Giovanna, Fastiggi Michele, Meneghelli Marta, Menzato Federica, Moschino Laura, Paladini Erika, Res Giulia, Roveran Martina, Zanella Sara, Zuccon Ilaria. Therapist Soravia Giulia, and nurses: Battistello Rachele, Biasin Elena, Bovo Lisa, Bregolin Marika, Cherubini Alessia, Chiapparino Anna Maria, Cocchiglia Laura, Colomba Antonina, Convento Giulia, Corbo Antonio, Costantini Anna, Dalla Montà Laura, Destro Giulia, Donolato Samantha, Foralosso Sabrina, Frasson Anna, Gibin Sabrina, Giuriato Ylenia, Greggio Silvia,
Heun Natascha, Lazzerini Lorena, Longo Marianna, Lucchin Marzia, Luise Chiara, Magnabosco Marta, Maglio Francesca, Manfrotto Valentina, Mastria Emanuel, Marzaloni Giulia, Mariniello Giovanna, Minelli Giovanna, Morettini Michele, Nigido Roberta, Norbiato Federica, Orzali Marika, Oselladore Giorgia, Passadore Alice, Pelizza Jacopo, Petrera Anna, Pescara Alessandra, Poggioli Giulia, Poli Giulia, Rampazzo Tatiana, Renesto Paola, Ricci Alice, Rosini Margherita, Rovoletto Federica, Salviato Alessia, Savastano Daniele, Sinigaglia Monica, Spata Monica, Tedeschi Elisabetta, Trevisan Tecla, Tombolato Maria, Varotto Alice, Vedolin Anna, Zamboni Jessica, Zanutto Alberta, Zinato Nicoletta, Zordan Francesca.

Authors' contributions Dr. Cavicchiolo and Dr Trevisanuto conceived the study and drafted the initial manuscript, in collaboration with Dr.Mardegan and Dr. Saieva. Mrs Lolli collected data and reviewed the manuscript. Prof. Plebani and Dr. Franchin contributed to prepare the protocol, provided relevant expertise, and critically reviewed the manuscript.

Prof. Baraldi conceived the study, reviewed and revised the manuscript in collaboration with Dr. Donato. All authors approved the final manuscript as submitted and agree to be accountable for all aspects of the work.

\section{Compliance with ethical standards}

Statement of ethics The study protocol complied with the Helsinki Declaration and was notified to the Institutional Ethical Committee in Padua (ref. number 00270821). Parents signed an informed consent form before the first specimen was collected.

Conflict of interest The authors declare that they have no conflicts of interest.

\section{References}

1. WHO. Coronavirus disease (COVID-2019) situation reports. Available at: https://www.who.int/emergencies/diseases/novelcoronavirus-2019/situation-reports. Accessed 14 June 2020

2. Rothe C, Schunk M, Sothmann P, Bretzel G, Froeschl G, Wallrauch C, Zimmer T, Thiel V, Janke C, Guggemos W, Seilmaier M, Drosten C, Vollmar P, Zwirglmaier K, Zange S, Wölfel R, Hoelscher M (2020) Transmission of 2019-nCoV infection from an asymptomatic contact in Germany. N Engl J Med 382(10): 970-971

3. Pan X, Chen D, Xia Y, Wu X, Li T, Ou X, Zhou L, Liu J (2020) Asymptomatic cases in a family cluster with SARS-CoV-2 infection. Lancet Infect Dis 20(4):410-411

4. Kampf G, Todt D, Pfaender S, Steinmann E (2020) Persistence of coronaviruses on inanimate surfaces and their inactivation with biocidal agents. J Hosp Infect 104(3):246-251

5. Zeng L, Xia S, Yuan W, Yan K, Xiao F, Shao J, Zhou W (2020) Neonatal early-onset infection with SARS-CoV-2 in 33 neonates born to mothers with COVID-19 in Wuhan, China. JAMA Pediatr 174:722. https://doi.org/10.1001/jamapediatrics.2020.0878

6. Dong Y, Mo X, Hu Y, Qi X, Jiang F, Jiang Z, Tong S (2020) Epidemiological characteristics of 2143 pediatric patients with 2019 coronavirus disease in China. Pediatrics 145:e20200702. https://doi.org/10.1542/peds.2020-0702

7. Vivanti AJ, Vauloup-Fellous C, Prevot S et al (2020) Transplacental transmission of SARS-CoV-2 infection. Nat Commun 11(1):3572 
8. Dong L, Tian J, He S et al (2020) Possible vertical transmission of SARS-CoV-2 from an infected mother to her newborn. JAMA. 323(18): $1846-1848$

9. Gagneur A (2008) Outbreaks of human coronavirus in a pediatric and neonatal intensive care unit. Eur J Pediatr 167(12):1427-1434

10. Ogimi J (2019) Characteristics and outcomes of coronavirus infection in children: the role of viral factors and an immunocompromised state. Pediatric Infect Dis Soc 8(1):21-28

11. Wang L, Shi Y, Xiao T et al (2020) Chinese expert consensus on the perinatal and neonatal management for the prevention and control of the 2019 novel coronavirus infection (First edition). Ann Transl Med 8(3):47

12. American Academy of Pediatrics. Management of infants born to mothers with COVID-19. Date of Document: April 2, 2020. Available at: https://aap.org

13. Trevisanuto D, Moschino L, Doglioni N et al (2020) Neonatal resuscitation where the mother has a suspected or confirmed novel coronavirus (SARS-CoV-2) infection: suggestion for a pragmatic action plan. Neonatology 1-8

14. De Luca D, Rava L, Nadel S et al (2020) The EPICENTRE (ESPNIC Covid pEdiatric Neonatal Registry) initiative: background and protocol for the international SARS-CoV-2 infections registry. Eur J Pediatr 22:1-8

15. Lavezzo E, Franchin E, Ciavarella C et al (2020) Suppression of a SARS-CoV-2 outbreak in the Italian municipality of Vo. Nature. 30

16. Cavicchiolo ME, Lolli E, Trevisanuto D et al (2020) Managing a tertiary-level NICU in the time of COVID-19: lessons learned from a high-risk zone. Pediatr Pulmonol:1-3

17. Interim guidelines for collecting, handling, and testing clinical specimens from persons for coronavirus disease 2019 (COVID-19). Accessed 14 June 2020. Available at: https://www.cdc.gov/ coronavirus/2019-ncov/lab/guidelines-clinical-specimens.html

18. Padoan A, Cosma C, Sciacovelli L, Faggian D, Plebani M (2020) Analytical performances of a chemiluminescence immunoassay for SARS-CoV-2 IgM/IgG and antibody kinetics. Clin Chem Lab Med. https://doi.org/10.1515/cclm-2020-0443

19. Cavicchiolo ME, Doglioni N, Ventola MA, Biban P, Baraldi E, Trevisanuto D (2020) Neonatal emergency transport system during
COVID-19 pandemic in Veneto region: proposal for standard operating procedures. Pediatr Res. https://doi.org/10.1038/s41390-0200937-z

20. Kimball A HK, Arons $M$ et al (2020) Asymptomatic and presymptomatic SARS-CoV-2 infections in residents of a long-term care skilled nursing facility — King County, Washington, March 2020. MMWR morbidity and mortality weekly report

21. Gandhi M, Yokoe DS, Havlir DV (2020) Asymptomatic transmission, the Achilles' heel of current strategies to control Covid-19. N Engl J Med 382(22):2158-2160

22. De Luca D (2020) Managing neonates with respiratory failure due to SARS-CoV-2. Lancet Child Adolesc Health 4(4):e8

23. Zaigham M, Andersson O (2020) Maternal and perinatal outcomes with COVID-19: a systematic review of 108 pregnancies. Acta Obstet Gynecol Scand 7

24. Gajbhiye RK, Modi DN, Mahale SD Pregnancy outcomes, newborn complications and maternal-fetal transmission of SARS-CoV2 in women with COVID-19: a systematic review. medRxiv preprint https://doi.org/10.1101/2020.04.11.20062356

25. Liguoro I, Pilotto C, Bonanni M, Ferrari ME, Pusiol A, Nocerino A, Vidal E, Cogo P (2020) SARS-COV-2 infection in children and newborns: a systematic review. Eur J Pediatr 179(7):1029-1046

26. Castagnoli R, Votto M, Licari A et al (2020) Severe acute respiratory syndrome coronavirus 2 (SARS-CoV-2) infection in children and adolescents. A systematic review. JAMA Pediatr 22

27. Elshafeey F, Magdi R, Hindi N et al (2020) A systematic scoping review of COVID-19 during pregnancy and childbirth. Int J Gynaecol Obstet 24

28. Food and drug aministration. Accessed 14 June 2020. Available at: https://www.fda.gov/medical-devices/emergency-situationsmedical-devices/faqs-diagnostic-testing-sars-cov-2\#serology

29. WHO. Laboratory testing for coronavirus disease (COVID-19) in suspected human cases: interim guidance, 19 March 2020. Available at: https://apps.who.int/iris/handle/10665/331329

Publisher's note Springer Nature remains neutral with regard to jurisdictional claims in published maps and institutional affiliations. 